\title{
Nonuniform Catalytic Behavior of Zeolite Crystals as Revealed by In Situ Optical Microspectroscopy**
}

\author{
Marianne H. F. Kox, Eli Stavitski, and Bert M. Weckhuysen*
}

In situ spectroscopic methods are essential to a fundamental understanding of catalytic reactions, thanks to their ability to unravel useful structure-function relationships. ${ }^{[1,2]}$ Recent developments have broadened the range of chemical reactions catalyzed by high-surface-area solids, which can be investigated in the act. ${ }^{[3-5]}$ However, most techniques average information over the whole catalyst sample, while in many cases it is of utmost importance to probe distinct areas of catalyst particles or grains to reveal how the structural features render into catalytic function. For this, microscopic methods may be of assistance. For example, IR and interference microscopy have been used to track the decomposition of template molecules, ${ }^{[6]}$ the orientation of adsorbates within porous catalytic solids, ${ }^{[7]}$ and the diffusion of organic compounds. ${ }^{[8-10]}$ Using fluorescence microscopy, the diffusion of organic chromophores in zeolites can be readily visualized on a microscopic scale. ${ }^{[11,12]}$ Furthermore, Raman and UV/Vis microspectroscopy have been exploited to investigate the mobility of metal-oxide species within catalyst extrudates during preparation. ${ }^{[13,14]}$ However, to the best of our knowledge, most of the above-mentioned techniques have not been applied directly to monitoring catalytic processes in a solid under true reaction conditions.

Herein, we investigate the oligomerization of styrene occurring in the micropores of ZSM-5 zeolite ${ }^{[15]}$ crystals by using in situ optical microspectroscopy. The cationic intermediates in this reaction, which exhibit strong optical absorption, can act as reporter molecules for catalytic activity. ${ }^{[16]}$ For this purpose, a novel in situ optical microspectroscopic setup has been developed in our laboratory. With this method, light is focused onto the specimen by a microscope objective, and the reflected photons are collected and directed to an optical fiber that is connected to a UV/Vis spectrometer. By scanning over the zeolite crystal, the spatially resolved optical absorption may be mapped with a spatial resolution down to several micrometers.

H-ZSM-5 crystals (Figure $1 \mathrm{a}$ ) exposed to 4-methoxystyrene were heated in situ in the spectroscopic cell, and images

[*] M. H. F. Kox, Dr. E. Stavitski, Prof. Dr. ir. B. M. Weckhuysen Inorganic Chemistry and Catalysis Group

Department of Chemistry

Utrecht University

Sorbonnelaan 16, 3584 CA Utrecht (The Netherlands)

Fax: $(+31)$ 30-251-1027

E-mail: b.m.weckhuysen@chem.uu.nl

$[* *$ We thank the Dutch National Science Foundation (NWO-CW VICI grant) and the Research School Combination Catalysis (NRSC-C) for financial support. Dr. Machteld Mertens (ExxonMobil, Machelen, Belgium) is acknowledged for providing the ZSM-5 crystals. We are grateful to Ingmar Swart for carrying out DFT calculations.
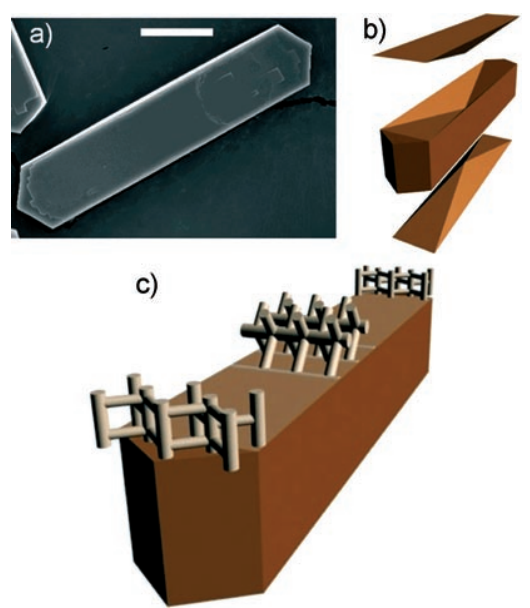

Figure 1. a) Scanning electron microscopy image of a ZSM-5 crystal $\left(\right.$ size $\left.100 \times 20 \times 20 \mu \mathrm{m}^{3}\right)$. Scale bar: $20 \mu \mathrm{m}$. b) "Exploded" representation of the crystal showing the individual building subunits. c) Zeolite channel alignment in the different regions of the crystal. The inner zeolite framework, which comprises intersecting straight and zigzag channels, is accessible from the outer surface through openings of the straight pores at the edges and zigzag pores at the body of the crystal.

and optical spectra were recorded. The crystals turn purple upon heating; however, closer examination reveals that the edges and main body of the crystal exhibit different colors (Figure 2a). As indicated by the optical spectra, two bands at $\approx 590$ and $635 \mathrm{~nm}$, present in both parts of the crystal with a different intensity ratio, are responsible for the observed color variations (Figure $2 \mathrm{~b}$ ). Along the lines of the reaction scheme presented in Figure $2 \mathrm{c},{ }^{[17]}$ the band at $590 \mathrm{~nm}$ arises from an allylic carbocation (Figure $2 \mathrm{c}$ ),${ }^{[16]}$ while the band at $635 \mathrm{~nm}$ has never been assigned. Nevertheless, it is evident that different parts of the crystal vary in their catalytic behavior.

To tackle the nonuniform catalytic properties of the ZSM5 crystal, deeper insight into its microscopic structure is required. ZSM-5 has the MFI crystalline structure with a twodimensional pore system, which consists of intersecting elliptical $5.3 \times 5.1-\AA$ straight pores and $5.6 \times 5.3-\AA$ zigzag pores (Figure 1c). ${ }^{[18]}$ Furthermore, large ZSM-5 crystals comprise three intergrown single-crystalline subunits (Figure $1 \mathrm{~b}),{ }^{[19,20]}$ rotated by $90^{\circ}$ about the common crystallographic [001] axis. As the oligomerization reaction is likely to occur in the zeolite channels, one would expect the carbocations to be entrapped and aligned within the pores. Such an alignment may be revealed by optical absorption measurements with polarized light, as the photophysically active molecules absorb light with the polarization parallel to their dipole moment vector. Microphotographs taken with nonpolarized and polarized light (Figure $3 \mathrm{a}-\mathrm{c}$ ) show that the 

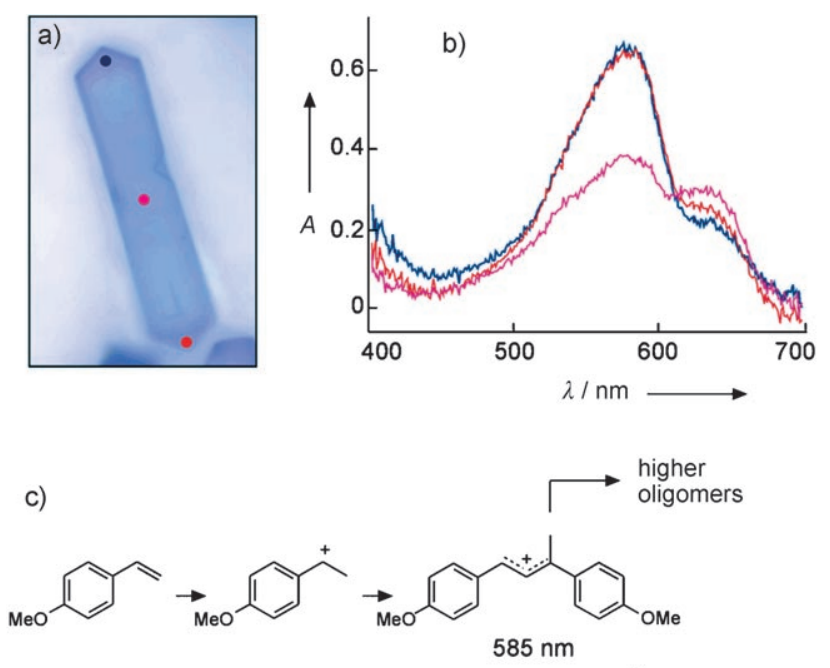

d)

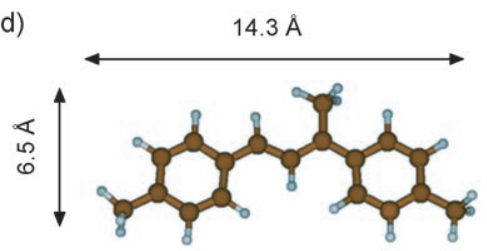

Figure 2. a) Optical microphotograph of a ZSM-5 crystal after addition of 4-methoxystyrene, which demonstrates the different colors observed at the body (blue) and edges (purple). b) Spatially resolved optical absorption spectra measured along the long axis of the crystal after reaction with 4-methoxystyrene at the edges (red and blue) and the center (pink). c) Reaction pathways of 4-methoxystyrene in the acidic zeolite; a neutral styrene molecule is protonated on a Brønsted acid site to form a carbocation, which reacts with another styrene molecule. The dimeric cation may undergo cyclization or react further. d) Calculated molecular geometry of the dimeric carbocation.

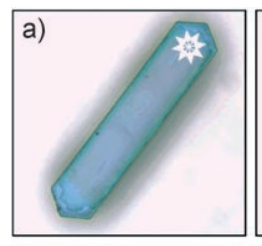

d)

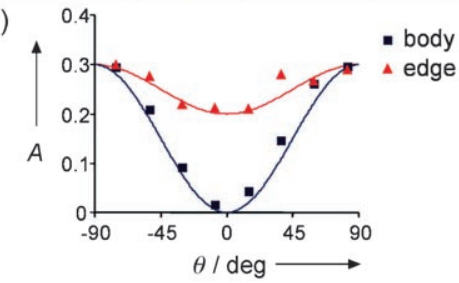

Figure 3. Microphotographs of $\mathrm{H}-\mathrm{ZSM}-5 / 4-m e t h o x y s t y r e n e$ taken with a) nonpolarized light (star) and b,c) polarized light; polarization is indicated by arrows. d) Angular dependence of the optical absorption at $590 \mathrm{~nm}$, at the body (blue) and edges (red) of the crystal, which follows the $\sin ^{2} \theta$ law, where $\theta$ is the angle between the light polarization vector and the long axis of the crystal.

body of the crystal (an upper surface of the smaller subunit) remains translucent when the polarization is parallel to the crystal long axis, but is strongly colored when the polarizer is directed otherwise. This finding directly implies that in this region the carbocation molecules are aligned with the straight channels (see Figure 1c). In principle, 6- $\AA$-thick dimer molecules may enter both the straight and zigzag channels; however, as the length of the segment of the latter equals $6.6 \AA$, a severely bent conformation should be imposed on the 14- $\AA$-long molecule. As for the edges of the crystal, upon rotation of the polarizer the absorbance does not change dramatically (Figure $3 \mathrm{~d}$, red line), which indicates that the preferred molecular orientation is collinear with the incident light, and makes $90^{\circ}$ angles with any light polarization.

Based on these findings, assignment of the band at $635 \mathrm{~nm}$ can be attempted. ${ }^{[21]}$ The shift to lower energies suggests an extended conjugated $\pi$ system; therefore, it is reasonable to assume that the band originates from trimeric carbocations. The observation that this band arises at later times compared to that from dimeric carbocations ${ }^{[22]}$ supports this assumption. Furthermore, as the absorbance at $635 \mathrm{~nm}$ is negligible at $0^{\circ}$, the band is likely to result from elongated molecules aligned in the straight channels. However, we could not rule out the possibility that the $635-\mathrm{nm}$ band arises from a different carbocation, that is, one derived from the cyclic structure (Figure 2c), although the following discussion is in line with the original assumption.

Given the band assignments, styrene oligomerization at the crystal edges appears to lead preferentially toward dimeric products, whereas in the main body both dimers and trimers are being formed in comparable quantities. As the rate-limiting step is the formation of the initial styrene carbocation, with the further steps being thermodynamically controlled, the difference in product formation at the edges and main body must be accounted for by diffusion limitations. The crystal structure (Figure 1c) suggests that at the edge regions straight pores, which accommodate the intermediate molecules, are opened directly to the outer surface, while in the main body they run parallel to the surface and are accessible through the sections of zigzag pores. Based on this structural information, a reaction mechanism may be put forward (Figure $4 a, b$ ). At the crystal edges, upon dimerization the carbocation is trapped within the straight pore near the surface, thus blocking further access of monomers and suppressing the formation of higher oligomers. In the case of the crystal body, even after the dimer has been formed in the straight channel, more monomer molecules can diffuse in through the sections of the adjacent zigzag pores, thereby interconnecting the straight pore with the surface.

To substantiate the proposed model, the concentration dependence of the optical spectra was examined (Figure $4 \mathrm{c}, \mathrm{d})$. One can see that at low concentration the edges exhibit exclusive selectivity toward dimer carbocations. At high concentration the straight channels are "overloaded" with reagent, which shifts the equilibrium slightly toward the trimer. The main body appears to be less reactive than the edges at low styrene concentration; however, as the amount of absorbed styrene increases, both bands intensify and match that at the edges. This observation supports the proposal that the zeolite network in the main body, accessible through the zigzag pore openings, provides reactant molecules with greater mobility. 
a)
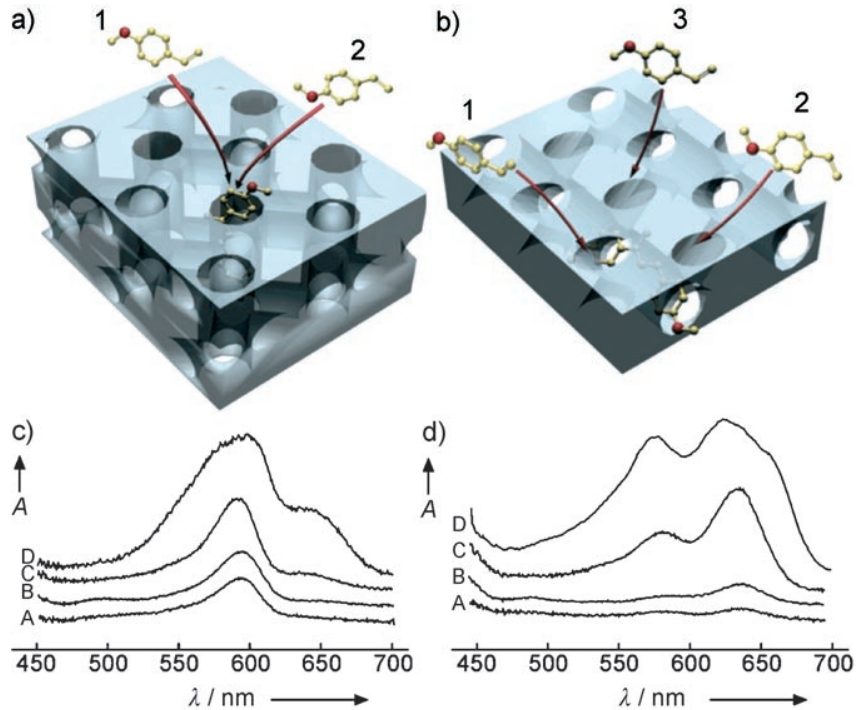

e)

b)

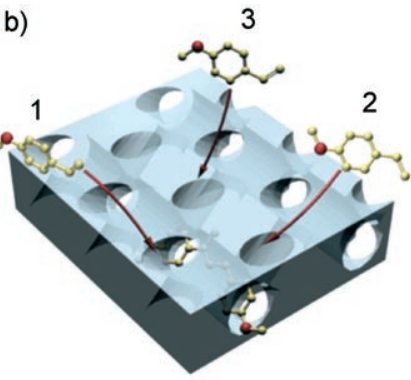

)

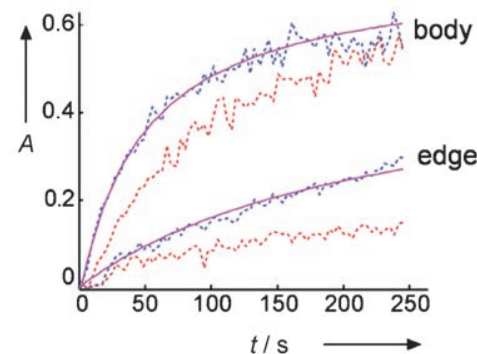

Figure 4. a) Reaction pathways at the edge region: upon formation of the dimerization product $(1+2)$, the straight pores are not accessible any more. b) The same as (a), but for the body region: zigzag pore openings adjacent to the reaction site provide access for monomer molecules (3) to diffuse inside and to react with the dimer. c,d) Concentration dependence of the absorption spectra at the edge and the body regions, respectively. The concentration of styrene in hexane solution is A) 36, B) 63 , C) 83 , and D) $100 \mathrm{wt} \%$. e) Time evolution of the optical absorption at $590 \mathrm{~nm}$ (blue) and $635 \mathrm{~nm}$ (red). The $590-\mathrm{nm}$ profiles are fitted using second-order kinetic equations (magenta) as described in the text.

The time evolution of the absorption spectra also verifies the proposed mechanism (Figure $4 \mathrm{e}$ ). The kinetics of dimer formation served as a measure of the catalytic activity. The formation rate was extracted by fitting the concentration profiles (intensities of the 590-nm band) using the secondorder kinetic equation $d=m_{0} /\left(1+m_{0} k t\right)$, where $m_{0}$ is the initial concentration of reagent, $d$ is the transient dimer concentration, and $k$ is the kinetic rate constant for a bimolecular process. As this simulation shows, dimer formation at the edges is slower $\left(k=0.02\right.$ versus $0.05 \mathrm{~mol}^{-1} \mathrm{~s}^{-1}$ for the body) as a result of blockage of the straight pore. Furthermore, the profile for the 635-nm band lags behind that of the 590-nm band, thus supporting its assignment to the formation of trimeric carbocations. ${ }^{[23]}$

It is tempting to compare the behavior of 4-methoxystyrene with that of other substituted styrene molecules, as it was found that a variety of related derivatives lead to the coloration of acidic zeolites. Our experiments have demon- strated that for all the compounds investigated (see Table 1), the general features of the optical spectra do not undergo significant changes, that is, the shorter-wavelength band assigned to the dimer predominates the edge spectrum,

Table 1: Reaction rate constants for the formation of styrene dimer at $353 \mathrm{~K}$.

\begin{tabular}{ll}
\hline Compound & $k\left[\mathrm{~mol}^{-1} \mathrm{~s}^{-1}\right]^{[\mathrm{a}]}$ \\
\hline 4-methoxystyrene (1) & 0.05 \\
4-ethoxystyrene (2) & 0.004 \\
4-methylstyrene (3) & 0.005 \\
4-bromostyrene (4) & 0.05 \\
4-chlorostyrene (5) & 0.009 \\
4-fluorostyrene (6) & 0.007 \\
$\beta$-methylstyrene (7) & 0
\end{tabular}

[a] The rate constants were obtained by fitting kinetic profiles using a second-order kinetic equation, as described in the text.

while the trimer-related longer-wavelength band prevails in the body. However, the temporal characteristics vary significantly (Table 1 ). To rationalize these results, two processes have to be taken into account in parallel: the diffusion of the reactant molecules within the channels and monomer protonation on the acid sites. The former depends mainly on the reactant kinetic diameter, whereas the latter is determined by the relative stability of the initial styrene carbocation. Along these lines, 4-ethoxystyrene (2) exhibits a reaction rate significantly lower than that of 4-methoxystyrene (1). As the carbocation stabilization properties of methoxy and ethoxy groups are expected to be similar, the diffusion slowdown as a result of the increase in molecular size accounts for this dramatic difference. On the other hand, an equally slower reaction rate for 4-methylstyrene (3) as compared to $\mathbf{1}$ is not because of geometric differences, as the methoxy moiety is bulkier than the methyl one. Instead, the methoxy group appears to provide a better means of carbocation stabilization through the formation of a resonative structure. In the row of halogenated styrenes, stronger electron-withdrawing groups (4-6) lead to lower styrene carbocation stability, as reflected by a decrease in the reaction rate in the row $\mathrm{Br}>\mathrm{Cl}>\mathrm{F}$. Finally, branched styrenes (7) do not react at all because of the steric hindrances in the channels. A thorough comparison of the oligomerization kinetics for a series of different styrene derivatives extending to a broader temperature range will be the subject of a subsequent publication.

In summary, a microspectroscopic investigation of the styrene oligomerization reaction in zeolite crystals allows valuable insights to be obtained into their catalytic performance. Although the spatial resolution of the method is in the micrometer range, we were able to deduce mechanistic features down to the molecular level. The results show how the diffusion and catalytic properties of straight and zigzag channels in ZSM-5 zeolite are combined in a synergistic catalytic action. We have also demonstrated that styrene chemistry in acidic zeolites sheds more light on the catalytic processes that occur within the zeolite channels. It is evident that the microspectroscopy approach may become a valuable 
asset in the arsenal of the catalysis scientist. In particular, the method can be readily adapted to gas-phase processes, as we were able to map the catalytic activity of ZSM-5 crystals in the methanol-to-hydrocarbon reaction.

\section{Experimental Section}

Materials: ZSM-5 crystals were provided by ExxonMobil (Machelen, Belgium). A Si/Al ratio of 17:1 was determined by X-ray fluorescence spectroscopy. Styrene derivatives (Acros Organics) were used as received.

Molecular geometry calculations: Density functional theory calculations of a styrene dimer carbocation were performed using the TURBOMOLE quantum chemistry package.

Microspectroscopic setup: The setup was based on an Olympus BX41 upright research microscope with a $50 \times, 0.5-\mathrm{NA}$ (NA: numerical aperature), high-working-distance microscope objective. A 75-W xenon lamp was used for illumination. The microscope was equipped with a 50/50 double-viewport tube, which accommodated a CCD video camera (ColorView IIIu, Soft Imaging System GmbH) and an optical fiber mount. A $200-\mu \mathrm{m}$-core fiber connected the microscope to a CCD UV/Vis spectrometer (AvaSpec-2048TEC, Avantes BV). A rotatable polarizer was introduced between the objective and detector to separate the desirable light polarization. An in situ cell (FTIR 600, Linkam Scientific Instruments) with a temperature controller (TMS 93) was used for thermal treatments.

Received: January 18, 2007

Published online: April 3, 2007

Keywords: microporous materials - oligomerization - styrene · UV/Vis spectroscopy $\cdot$ zeolites

[1] In Situ Spectroscopy in Heterogeneous Catalysis (Ed: J. F. Haw), Wiley-VCH, Weinheim, 2002.

[2] In Situ Spectroscopy of Catalysts (Ed: B. M. Weckhuysen), American Scientific Publishers, Stevenson Ranch, 2004.

[3] B. M. Weckhuysen, Nature 2006, 439, 548.

[4] S. J. Tinnemans, J. G. Mesu, K. Kervinen, T. Visser, T. A. Nijhuis, A. M. Beale, D. E. Keller, A. M. J. van der Eerden, B. M. Weckhuysen, Catal. Today 2006, 113, 3.
[5] M. B. J. Roeffaers, B. F. Sels, H. Uji-i, F. C. De Schryver, P. A. Jacobs, D. E. De Vos, J. Hofkens, Nature 2006, 439, 572.

[6] M. Nowotny, J. A. Lercher, H. Kessler, Zeolites 1991, 11, 454.

[7] F. Schüth, J. Phys. Chem. 1992, 96, 7493.

[8] G. Muller, T. Narbeshuber, G. Mirth, J. A. Lercher, J. Phys. Chem. 1994, 98, 7436.

[9] P. Kortunov, C. Chmelik, J. Karger, R. A. Rakoczy, D. M. Ruthven, Y. Traa, S. Vasenkov, J. Weitkamp, Adsorption 2005, $11,235$.

[10] J. Kärger, P. Kortunov, S. Vasenkov, L. Heinke, D. B. Shah, R. A. Rakoczy, Y. Traa, J. Weitkamp, Angew. Chem. 2006, 118, 8010; Angew. Chem. Int. Ed. 2006, 45, 7846.

[11] M. Pfenniger, G. Calzaferri, ChemPhysChem 2000, 1, 211.

[12] S. Hashimoto, K. Uehara, K. Sogawa, M. Takada, H. Fukumura, Phys. Chem. Chem. Phys. 2006, 8, 1451.

[13] L. G. van de Water, J. A. Bergwerff, T. A. Nijhuis, K. P. de Jong, B. M. Weckhuysen, J. Am. Chem. Soc. 2005, 127, 5024.

[14] J. A. Bergwerff, L. G. A. van de Water, T. Visser, P. de Peinder, B. R. G. Leliveld, K. P. de Jong, B. M. Weckhuysen, Chem. Eur. J. 2005, 11, 4591.

[15] A. Corma, Chem. Rev. 1995, 95, 559.

[16] F. L. Cozens, R. Bogdanova, M. Regimbald, H. Garcia, V. Marti, J. C. Scaiano, J. Phys. Chem. B 1997, 101, 6921.

[17] S. S. Pollack, R. F. Sprecher, E. A. Frommell, J. Mol. Catal. 1991, 66, 195.

[18] G. T. Kokotailo, S. L. Lawton, D. H. Olson, W. M. Meier, Nature 1978, 272, 437.

[19] G. D. Price, J. J. Pluth, J. V. Smith, J. M. Bennett, R. L. Patton, J. Am. Chem. Soc. 1982, 104, 5971.

[20] D. G. Hay, H. Jaeger, K. G. Wilshier, Zeolites 1990, 10, 571

[21] Although the band at $635 \mathrm{~nm}$ has been observed previously, ${ }^{[22]}$ its assignment has not yet been attempted.

[22] V. Fornes, H. Garcia, V. Marti, L. Fernandez, Tetrahedron 1998, $54,3827$.

[23] As the rate-limiting step of the oligomerization reaction is the formation of the primary styrene carbocation, a fitting using first-order kinetic curves has also been attempted. However, the second-order kinetics appears to fit the profiles more truthfully for the different reaction temperatures and all the compounds tested. 